\title{
PRODUCCIÓN DE EMBRIONES BOVINOS DEL LABORATORIO INVITRO COLOMBIA DURANTE EL AÑO 2019
}

\section{PRODUCTION OF BOVINE EMBRYOS FROM THE INVITRO COLOMBIA LABORATORY DURING 2019}

Lilian Bonilla León, Médico Veterinario Zootecnista, Esp. en Pedagogía, MsC en Ciencias Pecuarias

- Universidad Nacional Abierta y a Distancia.

ORCID: https://orcid.org/0000-0002-0841-051X

Correo: lilian.bonilla@unad.edu.co

Danilo Bonilla Trujillo, Médico Veterinario Zootecnista, Esp. en Pedagogía, MsC en Ciencias Pecuarias - Universidad Nacional Abierta y a Distancia.

ORCID: https://orcid.org/0000-0002-6069-8039

Correo: danilo.bonilla@unad.edu.co

Ramón Guillermo Gómez Domínguez, Médico Veterinario Zootecnista, Máster of business administration- INVITRO COLOMBIA SAS.

ORCID: https://orcid.org/0000-0002-4691-8787

Correo: ramón.gomez@invitro.com.co

Citación: Bonilla, L., Bonilla, D., y Gómez, R. (2021). Producción de embriones bovinos del laboratorio INVITRO COLOMBIA durante el año 2019. Working Papers ECAPMA, 1, 6 - 16. DOI: https://doi.org/10.22490/ECAPMA.4242

\section{RESUMEN}

Contextualización: La fecundación in vitro surgió hace 40 años, es una de las biotecnologías reproductivas que actualmente se están aplicando en el país, produciendo embriones comercialmente a gran escala en bovinos, la cual aumenta el aprovechamiento del potencial genético de la hembra.

Vacío del conocimiento: Colombia no presenta constantemente datos de producción ya que los laboratorios comerciales no publican sus resultados, 
evitando un posicionamiento del país en el mapa mundial de la biotecnología reproductiva aplicada a la producción comercial.

Propósito del estudio: La intención de este estudio es realizar un acercamiento a la producción promedio de un laboratorio dedicado a la producción de embriones in vitro en bovinos (PEIV) en Colombia.

Metodología: Se evaluó la producción de embriones del laboratorio INVITRO COLOMBIA SAS, del año 2019, en cuanto a la PEIV, teniendo en cuenta técnicas como la Aspiración folicular (OPU), la cual se puede realizar por la presencia de ondas foliculares del ciclo estral del bovino las cuales permiten que la colección de oocitos durante toda su vida productiva, Maduración in vitro (MIV), Fecundación in vitro (FIV) y Cultivo in vitro (CIV), 8 días después de la aspiración folicular son entregados los embriones producidos. Se evaluaron variables correspondientes a producción y preferencia racial y zootécnica en la aplicabilidad de la técnica por parte del productor. Para el análisis se utilizó estadística descriptiva por medio de la obtención de promedios.

Resultados y conclusiones: La producción de oocitos por donante es de 24 por sesión, la producción de embriones promedio fue de 4. La obtención de oocitos viables corresponde al $83.7 \%$, la división de zigotos fue de $66.5 \%$ y la producción de embriones a partir de los oocitos viables fue de $19.3 \%$. En cuanto a la vocación zootécnica de los animales sometidos a la técnica en un $76.4 \%$ corresponde a ganadería de leche y un $23.6 \%$ a ganadería de carne. La raza en la que se utiliza más la técnica es la raza Gyr, especialmente para cruces con razas europeas para la producción de F1 adaptados para ganadería de leche en el trópico, así como la Guzerat y la Brahman.

Palabras Claves: Aspiración folicular; fecundación in vitro; embriones; reproducción asistida en bovinos; biotecnología reproductiva

\section{ABSTRACT}

Contextualization: In vitro fertilization emerged 40 years ago; it is one of the reproductive biotechnologies that are currently being applied in the country, producing embryos commercially on a large scale in cattle, which increases the use of the genetic potential of the female.

Knowledge gap: Colombia does not constantly present production data since commercial laboratories do not publish their results, avoiding a positioning of the country in the world map of reproductive biotechnology applied to commercial production.

Purpose: The intention of this study is to make an approach to the average 
production of a laboratory dedicated to the production of bovine in vitro embryos (IVEP) in Colombia.

Methodology: The embryo production of INVITRO COLOMBIA SAS laboratory was evaluated for the year 2019, in terms of IVEP, taking into account techniques such as follicular aspiration (OPU), which can be performed by the presence of follicular waves of the bovine estrous cycle which allow the collection of oocytes throughout their productive life, in vitro maturation (IVM), in vitro fertilization (IVF) and in vitro culture (IVC), 8 days after follicular aspiration the embryos produced are delivered. Variables corresponding to production and racial and zootechnical preference in the applicability of the technique by the producer were evaluated. Descriptive statistics were used for the analysis by obtaining averages.

\section{INTRODUCCIÓN}

La fecundación in vitro surgió hace 40 años; desde la aplicación de la técnica en especies de producción hasta nuestros tiempos donde la producción comercial a larga escala se da, con resultados importantes para la mejora continua de las producciones pecuarias, no solo en animales de granja sino también en otras especies de interés
Results and conclusions: The production of oocytes per donor is 24 per session, the average embryo production was 4 . The obtaining of viable oocytes corresponds to $83.7 \%$, the division of zygotes was $66.5 \%$ and the production of embryos from viable oocytes was $19.3 \%$. Regarding the zootechnical vocation of the animals submitted to the technique, $76.4 \%$ corresponded to dairy cattle and $23.6 \%$ to beef cattle. The breed in which the technique is most used is the Gyr breed, especially for crossbreeding with European breeds for the production of F1 adapted for dairy cattle in the tropics, as well as the Guzerat and Brahman.

Keywords: Follicular aspiration; in vitro fertilization; embryos; bovine assisted reproduction; reproductive biotechnology.

comercial (Sirard, M. 2108). Si tenemos en cuenta que la fisiología normal del bovino en especial da paso a obtener una cría por año, para dar origen a la multiplicación de material genético de alta calidad se opta por técnicas de reproducción asistida que permita masificar el material genético (Plourde et al., 2012) y que el número de embriones producidos in vitro ha 
crecido exponencialmente a través de los últimos años, la cantidad de embriones producidos in vitro ha superado significativamente la de embriones in vivo, entonces la técnica se ha convertido en la opción más importante para los productores bovinos a nivel mundial (Sanches BV et al., 2019).

Las tecnologías de reproducción animal han sido utilizadas con éxito en todo el mundo, aumentan la importancia de las hembras en programas de mejoramiento genético (Guerreiro et al., 2014) y desempeñan un papel importante en la mejora de la precisión e intensidad de la selección de rasgos cuantitativos en vacas lecheras. Aproximadamente $\quad 1.275 .874$ embriones se produjeron a nivel mundial en 2013, 546.628 de los cuales fueron derivados de tecnologías in vitro (IETS (International Embryo Transfer Society), 2014). Brasil, es responsable del $70,8 \%$ del total de embriones in vitro producidos en el mundo en 2013 (IETS (International Embryo Transfer Society), 2014), la punción ovárica (OPU) seguida de la producción in vitro de embriones (PIV) es el principal procedimiento para aumentar el número de individuos de interés en el ganado Cebú (Perez et al., 2016)

Este estudio se enfoca en el análisis de producción del laboratorio de PEIV, con el fin de dar a conocer como son los resultados obtenidos de la práctica comercial de la reproducción asistida en bovinos, analizando el componente racial y la vocación zootécnica de los embriones.

\section{METODOLOGÍA}

\section{Técnica de aspiración folicular y fecundación in vitro}

Las ondas de crecimiento folicular en bovinos se caracterizan por el crecimiento, (2 -3 días) de 8 - 41 folículos (promedio 24), detectados por ultrasonografía, los cuales miden de 2 $4 \mathrm{~mm}$ de diámetro. En la última onda de crecimiento folicular, es seleccionado un folículo que continua el crecimiento y alcanza la dominancia mientras que los subordinados sufren atresia. Más del $95 \%$ de los ciclos estrales en ganado se componen de 2 a 3 ondas de crecimiento folicular. La emergencia de la primera onda folicular comienza el día de la ovulación (día 0), la segunda onda folicular emerge a los 9 o 10 días en ciclos de dos ondas y en el día 8 o 9 en ciclos de tres ondas (es decir 1 o 2 días antes). En ciclos de tres ondas la última onda emerge el día 15 - 16. Bajo la influencia de la progesterona, los folículos dominantes de las subsecuentes ondas permanecen anovulatorios y sufren atresia. El folículo dominante al inicio de la luteólisis, se convierte en el folículo ovulatorio, y la aparición de la siguiente onda se retrasa hasta el día de la siguiente ovulación. El surgimiento de 
una onda preovulatoria de $\mathrm{FSH}$ es seguida inmediatamente por otra distinta onda preovularoria la cual da origen a la emergencia de la primera onda del ciclo (Ginther et al., 2013)

El cuerpo lúteo comienza a retroceder antes en ciclos de dos ondas (día 16) que en ciclos de tres ondas (día 19), resultando en un ciclo estral más corto (20 y 23 días respectivamente), por lo tanto, un ciclo estral de 21 días es simplemente un promedio entre ciclos de 2 y 3 ondas de intervalos interovulatorios (Adams et al., 2012).

La fertilización in vitro es una técnica que facilita la unión de los gametos masculinos y femeninos para formar un cigoto en condiciones de laboratorio; el esperma seleccionado es capacitado y se co-incuba con el complejo cumulusovocitos maduros (Machaty y Peippo, 2012).

Aspiración Folicular (OPU): Las heces son retiradas del recto y la zona perineal se limpia con agua y Etanol al $70 \%$. Antes de la OPU, cada vaca recibe anestesia epidural $(5 \mathrm{ml}$ de lidocaína al 2\%; anestésico) para disminuir el peristaltismo y el malestar (Morotti et al., 2014). Utilizando un ecógrafo veterinario de modo B a tiempo real, con un transductor mini convex de $7.5 \mathrm{MHz}$, se adecua a un dispositivo intravaginal con una guía de acero inoxidable, se usa una aguja descartable de 19G x 1.5" (Morotti et al., 2014), la cual va unida por un sistema de conducción a un tubo cónico de $50 \mathrm{ml}$, para así llevar a cabo la punción del ovario. La punción se realiza con una presión negativa de 65 - 75 Hgmm, con una bomba de vacío. El líquido folicular y los oocitos caerán en una solución de medio TCM 199, con 25mM Hepes buffer, $5 \%$ de suero fetal bovino, $50 u$ de sulfato de gentamicina y $10.000 \mathrm{UI} / \mathrm{L}$ de Heparina Sódica (Seneda et al., 2001).

\section{Producción de embriones in vitro (PEIV)}

Maduración In Vitro (MIV): En el proceso In-Vivo la maduración del ovocito ocurre dentro del folículo y es conducido por eventos locales y sistémicos que requieren interacción entre el oocito y las células somáticas al rededor. Dado el desafío en el cual están involucrados los oocitos madurados in vivo muestran mayor capacidad de desarrollo que sus contrapartes in vitro. Cierto ARN mensajero y patrones de expresión de proteínas determinan la calidad de los oocitos. Se conoce que las condiciones de CIV utilizadas para la maduración de ovocitos dependen de la expresión genética $y$, por lo tanto, la capacidad de desarrollo. Además, si bien se han optimizado los medios de maduración rara vez se producen más del $30 \%$ al $40 \%$ de tasas de 
blastocitos, en comparación con tasas casi dos veces superiores obtenidas Con ovocitos madurados in vivo bajo la misma fertilización. (Nogueira et al., 2016).

- Fertilización In Vitro (FIV). La mayoría de los ovocitos son penetrados por el espermatozoide dentro de 4-6 horas con formación de pronúcleo entre 6 - 10 horas después adición de espermatozoides (Leibfried-Rutledge, et al., 1989; Eid LN et al., 1994). Cambiar la cantidad de esperma añadido al sistema de fertilización in vitro bovino también afecta la fertilización (First y Parrish, 1988; Parrish y Eid, 1994). Cuando las tasas de fertilización superan el $80 \%$, la polispermia aumenta, afectando las tasas de desarrollo.

- Cultivo in vitro (CIV): 18 h después de la fertilización, los cigotos son despojados de las células del cúmulo. Grupos de presuntos zigotos son cocultivados en una monocapa de células del cúmulus. El medio MIV es reemplazado por $50 \mathrm{ml}$ de medio CR2aa suplementado con $2 \%$ de FCS y $30 \mathrm{mg} / \mathrm{ml}$ BSA, y los embriones se cultivan a $39^{\circ} \mathrm{C}$ en humidificación de aire con $5 \%$ de $\mathrm{CO} 2$ durante 48 a 72 $h$, momento en el que $30 \mathrm{ml}$ de medio fresco de cultivo se añade (primera alimentación). La tasa de división embrionaria o clivaje se registra después de 3 días de cultivo.
La segunda alimentación se realiza el sexto día, y la tasa de blastocitos (el número total de blastocistos dividido por el número total de oocitos cultivados) se registra en el séptimo día de cultivo de embriones. (Guerreiro et al., 2014)

\section{MATERIALES Y MÉTODOS}

\section{ANÁLISIS DE LOS RESULTADOS}

Para el análisis se aplicó estadística descriptiva usando la media como medida de tendencia central la cual permitió evaluar la producción de embriones del laboratorio IN VITRO COLOMBIA SAS, durante el año 2019, determinando los totales de producción, teniendo en cuenta variables como: total de donantes, total de sesiones de aspiración, promedio de donantes por sesión de aspiración, oocitos totales, promedio oocitos por sesión de aspiración, oocitos viables, clivaje o división embrionaria, total de embriones producidos, promedio de embriones producidos por sesión y promedio de embriones producidos por vaca. Además, se estableció la preferencia de la raza de la donadora para ser sometida a la técnica, determinando el porcentaje de animales de cada raza utilizados para el procedimiento, así como la vocación zootécnica de los cruces a obtener del proceso. 
Los resultados de producción obtenidos incluyen los valores de cero, donde no hubo producción de embriones por factores que pueden afectar la producción como: contaminación, estado sanitario del animal, calidad seminal o problemas asociados a la logística del laboratorio.

Se debe tener en cuenta que en algunos procedimientos se utiliza semen sexado, el cual tiene un desempeño menor en producción que el semen convencional, disminuyendo de manera significativa los promedios que con el uso de semen convencional se podrían obtener (Bonilla et al, 2018).

\section{DISCUSIóN}

Se observa una producción de embriones a gran escala, la cual impacta de positivamente el mejoramiento genético del país. Entonces, el promedio de producción de oocitos por donante por sesión de aspiración es de 24, la cantidad de donantes aspiradas por sesión en promedio es de 6 y la cantidad de embriones producidos por hembra es de 4.

El porcentaje de oocitos viables del total de los aspirados es del $83.7 \%$. El porcentaje de cigotos divididos luego de la fecundación es de $66.5 \%$, La producción de embriones totales respecto a los oocitos viables es de $19.3 \%$. La producción general obtenida se encuentra en la tabla 1.

Tabla 1. Producción de embriones In Vitro (PIV).

\begin{tabular}{cc} 
Procedimiento & Totales \\
Sesiones de aspiración folicular & 1.142 \\
Oocitos totales obtenidos & 163.566 \\
Total donantes & 6.795 \\
Oocitos viables & 136.949 \\
Zigotos Divididos & 91.054 \\
Total Embriones producidos & 26.424 \\
\hline
\end{tabular}

Fuente: Autores.

Según el tipo racial se observa la importancia que la raza Gyr tiene en el país, ya que el $62.74 \%$ de la producción del laboratorio, corresponde a donantes de esta raza. En esta producción se incluyen los cruces puros y cruces con Bos Taurus.
1. Preferencia Racial de las donantes sometidas al proceso de PIV: en la gráfica 2, se observa la distribución de las donantes sometidas a PEIV, según su tipo racial: 


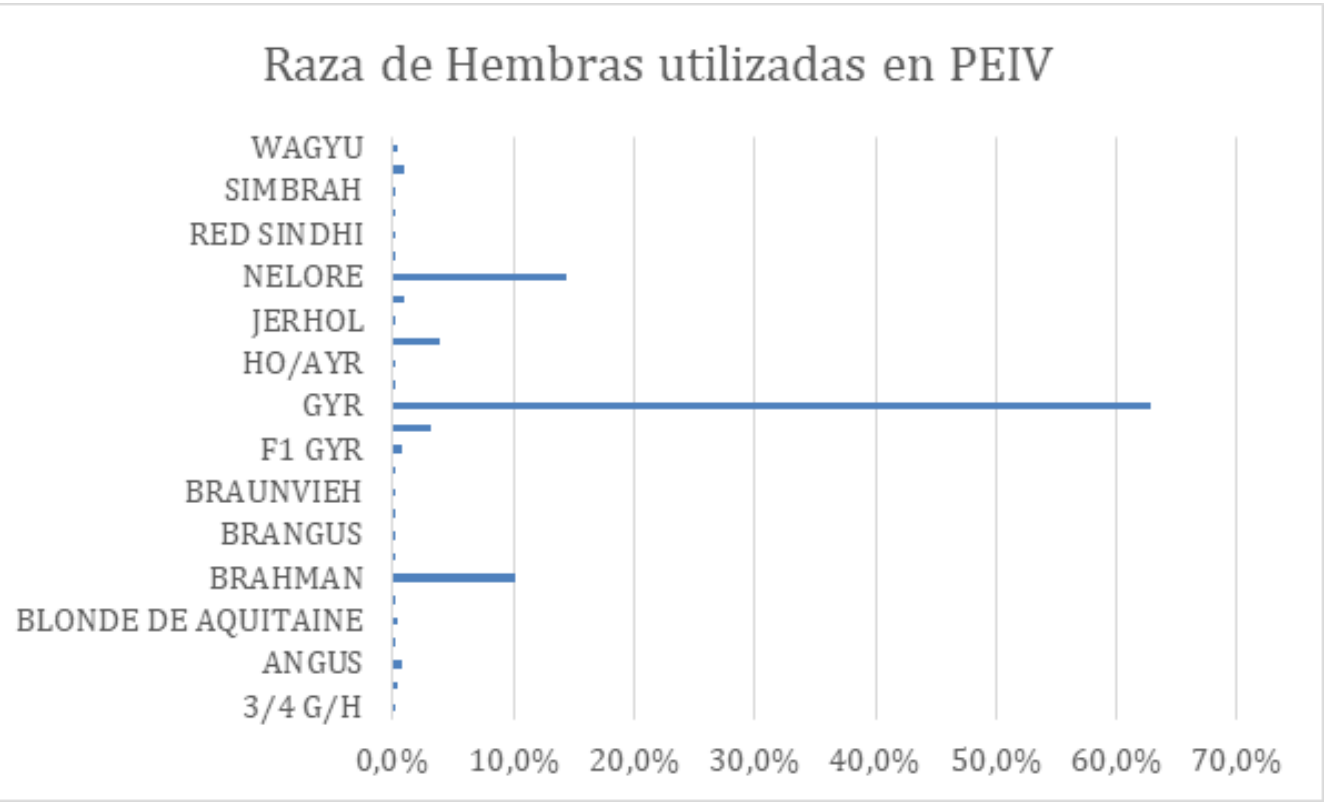

Grafica 1. Distribución de hembras sometidas a PEIV, según su tipo racial. Fuente: autores.

El segundo lugar con un $14.39 \%$ lo ocupa la raza Nelore. Las razas Brahman y Guzetat, se posicionan con $\quad 10.23 \% \quad y \quad 3.05 \%$ respectivamente, dejando ver que las principales razas a las cuales se les aplica esta técnica de reproducción asistida corresponden a la subespecie Bos indicus.

La única raza Bos Taurus con una participación importante en la producción es la raza Holstein con un $3.88 \%$, utilizada en especial para cruces con toros Bos indicus.

2. Vocación zootécnica general de los embriones producidos in vitro: de las 6795 hembras sometidas al proceso de PEIV, se definió que el $76.4 \%$ corresponde a ganadería de leche y el $23.6 \%$ a ganadería de carne.

3. Cruces Bos Taurus $x$ Bos indicus: la preferencia de la técnica para los cruces Bos Taurus x Bos Indicus se observan en la tabla 2. Para estos cruces se opta por utilizar donadoras Bos indicus, que incluyen las razas: Gyr, Guzetat y considerando la raza Brahman perteneciente a esta subespecie, con el uso de razas europeas de lechería especializada, con toros probados o genómicos. Se observa la utilización de semen sexado, para asegurar en un mayor porcentaje el nacimiento de hembras para minimizar las perdidas por nacimiento de machos en las explotaciones lecheras del país. 
Tabla 2. Distribución de razas de toros Bos taurus usados en PEIV en cruces con donantes Bos indicus.

\begin{tabular}{|c|c|c|c|c|c|c|}
\hline \multicolumn{2}{|c|}{ Raza de la donante } & \multicolumn{4}{c|}{ Raza del toro } \\
\hline $\begin{array}{c}\text { Cruce } \\
\text { para } \\
\text { lechería }\end{array}$ & $53.58 \%$ & $\begin{array}{c}\text { Pardo } \\
\text { Suizo }\end{array}$ & Holstein & Jersey & Simmental \\
\hline GYR & 3328 & $91,55 \%$ & 89 & 3221 & 13 & 5 \\
\hline BRAHMAN & 149 & $4,10 \%$ & - & 149 & - & - \\
\hline GUZERAT & 158 & $4,35 \%$ & - & 140 & 18 & - \\
\hline $\begin{array}{c}\text { Total } \\
\text { por Raza }\end{array}$ & 3635 & & 89 & 3510 & 31 & 5 \\
\hline \multicolumn{2}{|c|}{ Porcentaje por raza } & $2,4 \%$ & $96,6 \%$ & $0,9 \%$ & $0,1 \%$ \\
\hline
\end{tabular}

Fuente: Autores.

\section{CONCLUSIONES}

La ganadería de leche es la explotación que más invierte en mejoramiento genético, como las técnicas de reproducción asistida, en este caso la PEIV.

La raza Gyr es la raza más utilizada en PEIV, en especial para la obtención de hembras de remplazo en las explotaciones, por medio del uso sexado de razas europeas.

La aplicación de técnicas de reproducción asistida, cada vez están al alcance de los productores, en especial la PEIV, que es una técnica fácil de implementar logística y económicamente.

\section{REFERENCIAS}

Adams, G. P., Singh, J., \& Baerwald, A. R. (2012). Large animal models for the study of ovarian follicular dynamics in women. Theriogenology, 78(8), 17331748.

https://doi.org/10.1016/j.theriogenolog y.2012.04.010

Bonilla León, L., Mejía Gallego, A., Gómez Domínguez, R., Torres Londoño, M., \& Uribe García, F. (2018). Viabilidad y tasa de preñez de embriones producidos in vitro a partir de semen sexado comparado con semen convencional en Bos taurus y Bos indicus. Revista de Investigaciones Veterinarias del Perú, 29(4), 1377-1385. https://dx.doi.org/10.15381/rivep.v29i $\underline{4.14297}$

da Costa, N. N., Brito, K. N., Santana, P. d., Cordeiro, M., Silva, T. V., Santos, A. X., Ramos, P., Santos, S., King, W. A., 
Miranda, M., \& Ohashi, O. M. (2016). Effect of cortisol on bovine oocyte maturation and embryo development in vitro. Theriogenology, 85(2), 323-329. https://doi.org/10.1016/j.theriogenolog y.2015.08.010

Eid, L.N., Lorton, S. P., \& Parrish, J.J. (1994). Paternal Influence on S-Phase in the First Cell Cycle of the Bovine Embryo. Biology of Reproduction, Volume 51, Issue 6, 1 December 1994, Pages 1232-1237, https://doi.org/10.1095/biolreprod51.6. 1232

First, N.L.; Parrish, J.J. (1988). Sperm Maturation and In Vitro Fertilization. The 11th Int. Congress on Reprod. A.I; Dublin, Ireland. p. 160-8. https://catalog.hathitrust.org/Record/1 $\underline{02255802}$

Ginther, O.J., Pinaffi, F.L., Khan, F.A., Duarte, L.F., \& Beg, M.A. (2013). Follicular-phase concentrations of progesterone, estradiol-17b, LH, FSH, and a PGF2a metabolite and daily clustering of prolactin pulses, based on hourly blood sampling and hourly detection of ovulation in heifers. Theriogenology, 79, 918-928 https://doi.org/10.1016/j.theriogenolog y.2012.12.015

Guerreiro, B. M., Batista, E. O., Vieira, L. M., Sá Filho, M. F., Rodrigues, C. A., Castro Netto, A., Silveira, C. R., Bayeux, B. M., Dias, E. A., Monteiro, F. M., Accorsi, M., Lopes, R. N., \& Baruselli, P. S. (2014). Plasma anti-mullerian hormone: an endocrine marker for in vitro embryo production from Bos taurus and Bos indicus donors. Domestic animal endocrinology,

49,

96-104. https://doi.org/10.1016/j.domaniend.2 $\underline{014.07 .002}$

International Embryo Transfer Society (IETS). (2014). Statistics of embryo collection in farm animals. https://www.iets.org/pdf/comm data/D ecember2015.pdf

Leibfried-Rutledge, M.L., Critser, E.S., Parrish, J.J., \& First, N.L. (1989). In vitro maturation and fertilization of bovine oocytes. Theriogenology; 31:61-74. https://doi.org/10.1016/0093$\underline{691 \times(89) 90564-5}$

Machaty, Z., Peippo, J., \& Peter, A. (2012). Production and manipulation of bovine embryos: techniques and terminology. Theriogenology, 78(5), 937-950.

https://doi.org/10.1016/j.theriogenology. $\underline{2012.04 .003}$

Morotti, F., Sanches, B. V., Pontes, J. H., Basso, A. C., Siqueira, E. R., Lisboa, L. A., \& Seneda, M. M. (2014). Pregnancy rate and birth rate of calves from a large-scale IVF program using reversesorted semen in Bos indicus, Bos indicus-taurus, and Bos taurus cattle. Theriogenology, 81(5), 696-701. https://doi.org/10.1016/j.theriogenolog y.2013.12.002

Perez, B. C., Peixoto, M. G., Bruneli, F. T., Ramos, P. V., \& Balieiro, J. C. (2016). Genetic analysis of oocyte and embryo production traits in Guzerá breed donors and their associations with age at first calving. Genetics and molecular research: GMR, 15(2), 10.4238/gmr.15027583. https://doi.org/10.4238/gmr.15027583

Plourde, D., Vigneault, C., Laflamme, I., Blondin, P., \& Robert, C. (2012). Cellular and molecular characterization of the 
impact of laboratory setup on bovine in vitro embryo production. Theriogenology 77: 1767-1778. doi: $10.1016 / \mathrm{j}$.theriogenology. 2011.12.021. https://doi.org/10.1016/j.theriogenolog y.2011.12.021

Sanches, B. V., Zangirolamo, A. F., \& Seneda, M. M. (2019). Intensive use of IVF by large-scale dairy programs. Animal Reproduction, 16(3), 394-401. Epub November 28, 2019. https://doi.org/10.21451/1984-3143ar2019-0058

Seneda, M. M., Esper, C. R., Garcia, J. M., Oliveira, J. A., \& Vantini, R. (2001). Relationship between follicle size and ultrasound-guided transvaginal oocyte recovery. Animal reproduction science, 67(1-2), 37-43.

https://doi.org/10.1016/s0378-

4320(01)00113-0

\section{(c) $(9)(2)$}

